\title{
La calceta de plátano como materia prima en la implementación de elementos deportivos
}

\section{Plantain Leaf Fiber as a Raw Material for Sports Items}

Andres Felipe Portillo Tovar ${ }^{4}$ y Brayan Steven Arias Murcia ${ }^{5}$

\section{Resumen}

Este trabajo relacionó la reutilización de la calceta de plátano en la fabricación de elementos deportivos con el cuidado del medio ambiente, para estimular el uso de elementos biodegradables y disminuir así la cantidad de residuos desechados. Se hizo previamente una exploración bibliográfica acerca de los productos que se han realizado con dicho material, la cual ayudó en la orientación del documento. Luego, se realizó una encuesta para determinar el nivel de aceptación en la población escogida, evidenciando que el 79,4 \% no conocía estos productos alternos, lo que permitió que la población reflexionara sobre la reducción de elementos contaminantes. Por último, se concluyó que de materializarse dicho proyecto, sería un acierto tanto en el ámbito de innovación en la construcción de materiales de entrenamiento físico, como para estimular el uso de elementos ecoamigables.

Palabras clave: aprovechamiento de material orgánico, protección ambiental, elementos deportivos biodegradables.

\section{Abstract}

This work associated the reuse of plantain leaf fiber in manufacturing sports items to caring for the environment, thus stimulating the incorporation of biodegradable ele-

\footnotetext{
4 Estudiante Profesional de Entrenamiento Deportivo, Fundación Universitaria del Área Andina. Correo: aportillo3ßestudiantes.areandina.edu.co

5 Estudiante Profesional de Entrenamiento Deportivo, Fundación Universitaria del Área Andina.

Correo: barias20Restudiantes.areandina.edu.co
} 
ments and reducing waste. The literature was previously reviewed to determine the products made with this material. A survey was carried out to determine the level of acceptance in the chosen population, finding that $79.4 \%$ did not know these alternative products. Thus, we made the population reflect on the reduction of polluting elements. Finally, we concluded that if this project were to materialize, it would succeed in making innovative sports items and stimulating the use of eco-friendly products.

Keywords: Use of organic material, Environmental protection, Biodegradable sports items.

\section{Introducción}

El presente artículo hace referencia al uso de la calceta de plátano, específicamente desde el acondicionamiento físico, para exponer la forma en la que se utilizará esta materia prima en la fabricación de implementos deportivos. Esto, a su vez, reducirá la utilización de plásticos y la contaminación en el medio ambiente. La problemática que existe en algunos departamentos de Colombia respecto a la producción de plátano es desbordante. Por tanto, genera mucho residuo, el cual se puede aprovechar para darle un uso sostenible. Este tema tiene relevancia en nuestro contexto social, ya que el cambio climático, la contaminación ambiental y los movimientos ambientalistas son un tema bastante importante en este momento para el país y para el mundo. Científicamente la relevancia que tiene este residuo es que su tratamiento químico permitirá producir un material biodegradable, para que así se puedan fabricar implementos deportivos con una vida útil que no afectará ambientalmente al planeta.

El desarrollo de este artículo tiene como objetivo categorizar las propiedades de la calceta de plátano y los beneficios de su uso en la fabricación de elementos deportivos para preservar y aprovechar los recursos no renovables en el bienestar del ser humano y del planeta. Este residuo, al ser transformado por procesos químicos, crea un material a base de un polímero biodegradable que puede sustituir los materiales contaminantes como el plástico en la elaboración y comercialización de elementos deportivos.

Anteriormente se han desarrollado proyectos basados en el cuidado y sostenibilidad del medio ambiente, los cuales han tenido receptividad con el fin de reducir la huella ambiental. Con este se pretende involucrar a los diferentes clu- 
bes, escuelas e instituciones. Por ello, es trascendente visualizar las ventajas en su implementación y comercialización en productos deportivos.

El contenido temático desarrollado inicia con el planteamiento de la problemática y objeto del proyecto, concebido como el uso de la caleta de plátano y su reuso en la producción ecoambiental de artículos deportivos. Luego, se presentan autores de publicaciones que exponen temáticas sobre la materia prima empleada y enfatizan en la fabricación y creación de nuevos materiales. Enseguida, continúa con el marco teórico estructurado en aspectos como: La calceta de plátano, Beneficios de la calceta de plátano, Procesos en la producción de la calceta de plátano y banano e Impacto Ambiental, llegando a la exposición de la metodología basada en una encuesta con ítems enfocados en identificar las preferencias de las personas frente a los productos hechos con material biodegradable, el conocimiento del tema y sus distintas aplicaciones, lo cual dio pie a la lectura e interpretación de los resultados hacia la exteriorización de las conclusiones derivadas.

\section{Antecedentes}

Según Velastegui et al., (2017) en el artículo Análisis sobre el aprovechamien- to de los residuos del plátano, como materia prima para la producción de materiales plásticos biodegradables muestra cómo los residuos orgánicos producidos en los cultivos plataneros no son aprovechados correctamente debido al miedo de los productores al implementar nuevas tecnologías, pensando en que estas puedan ser un factor que afecte la economía de su empresa. El autor, además, comenta que de dichos cultivos solo el $5 \%$ representa el fruto que es aprovechado, el otro $95 \%$ se ha convertido en un factor contaminante del medio ambiente. Igualmente, hace referencia a una encuesta realizada en Guayaquil a 91 empresas productoras de plástico donde busca concientizar acerca de la importancia de repensar las materias primas hacia el uso de materiales amigables con el medioambiente. Los resultados de dicha encuesta fueron, el $66 \%$ reconoce que debe implementar el aprovechamiento de estos residuos, mientras que el $34 \%$ restante está en contra de dichas medidas.

Valenzuela et al., (2019) en su artículo Determination of Plantain Crops Associated With Coffee Environmental Impacts on Agroecosystems by Means of Life Cycle Assessment: Case Study in the Southwest of Antioquia (Colombia), da a conocer la carga del impacto ambiental 
de las hojas de plátano con relación a las hojas de café en algunas fincas de antioquia, para lo cual realizaron encuestas y entrevistas a los productores de la región. Además, muestra las cuatro etapas del ciclo de obtención del plátano las cuales son: establecimiento, producción, poscosecha y distribución, lo que evidencia un alto impacto ambiental en las etapas de producción (fabricación) debido al alto uso de fertilizantes cargados de potasio, nitrógeno y fósforo. A partir de esto se concluye que no son pertinentes las prácticas ambientales y agrícolas utilizadas.

Alarcon et al., (2019) en su publicación Producción y comercialización de platos descartables y biodegradables hechos a base de papel kraft y hojas de plátano, describe el proyecto realizado en Perú, el cual busca encontrar en la hoja de plátano la materia prima para la elaboración de platos amigables con el medio ambiente y, a su vez, disminuir el nivel de contaminación. Por otra parte se utilizaron entrevistas para determinar el nivel de aceptación por el público, dando como resultado un producto final con las características esperadas.

Pedraza (2019), en el proyecto de investigación sobre la Caracterización de la fibra del pseudotallo de plátano como refuerzo y desarrollo de un material com- puesto para fabricación de tejas, desarrollado en Duitama, Colombia, muestra la importancia de darle un uso a la calceta de plátano ya que en el país se explota el producto. Este se puede implementar en la fabricación de tejas con un material compuesto que es desarrollado a partir de tejidos simples y cruzados, expuestos a ensayos de flexión y compresión. Dicho material se obtiene por medio de un método óptimo de extracción de la fibra de pseudotallo de plátano, después de ser usadas varias técnicas para la obtención de las mismas, logrando como resultado fibras de buena calidad y longitudes y grosores similares. El 50,7 \% del total del área agrícola cosechada en el área rural colombiana corresponde a cultivos de plátano, donde la producción del mismo presenta la mayor participación con el $37,3 \%$ de toneladas. Si se tiene en cuenta que de la planta del plátano solo el $12 \%$ se considera comerciable (fruto) y el 88 $\%$ restante se estima residuo, es mucho lo que se aprovecharía como materia prima para este proyecto.

\section{Marco teórico}

\section{La calceta de plátano y sus beneficios}

Rodríguez et al., (2017) definen la calceta de plátano —o también llamado guas- 
ca de plátano - como una planta herbácea que tiende a ser de gran tamaño. Alcanza alrededor de $7 \mathrm{~m}$ de altura y carece de un verdadero tronco. Esta se conoce por ser una fibra que proviene de la corteza de la mata o de las hojas del tallo, también conocidas como pseudotallo. La disponibilidad de esta materia prima proviene de los cultivos que se generan en el territorio colombiano; siendo a su vez un material orgánico o residual poco aprovechado.

La calceta de plátano es la materia prima para elaborar diferentes productos artesanales como: individuales, cofres, tapetes, tapices, telas, entre otros. Por esta razón es importante poner en marcha diferentes programas y medios tecnológicos agrícolas para la ampliación en el cultivo de este producto. Los principales beneficios de la calceta de plátano son su biodegradación, que a su vez aporta al cuidado del medio ambiente y promueve el desarrollo sostenible colaborando en el aumento económico, el progreso social y la estabilización ambiental. Alarcón, et al (2019).

\section{Procesos en la producción de la calceta de plátano y banano}

Rodríguez, et al (2017) describen el proceso de producción de plátano, cortando el tallo a $40 \mathrm{~cm}$, evitando afectar las plantas hijas que puedan brotar. Después se posicionan verticalmente las hojas y el tallo y se dejan durante 24 horas para que la sábila salga y se pueda secar sin que la calceta se descomponga. Luego, se retiran las mismas con extremada delicadeza para no afectar su estructura. Enseguida, se procede a colocarlas hacia abajo dejándolas encima de láminas de zinc y expuestas al sol por tres días, hasta que tornen un color marrón y estén totalmente secas. Se continúa con un proceso de selección en donde se tiene en cuenta tamaño, color y calidad. Posteriormente se hace un proceso de extracción de las fibras o filamentos, para luego realizar un proceso de limpieza y enjuague.

\section{Impacto ambiental}

Valenzuela et al., (2019) describen en su artículo Determination of Plantain Crops Associated with Coffee Environmental Impacts on Agroecosystems by Means of Life Cycle Assessment: Case Study in the Southwest of Antioquia (Colombia), el proceso relevante trabajado en su estudio para la obtención del plátano, bajo cuatro etapas las cuales son:

- Establecimiento: en esta fase son necesarios recursos como el fertilizante, los desinfectantes, los correctivos, el combustible para el transporte y el 
manejo del equipamiento para poder instalar los cultivos a tratar. Además, fue necesario determinar el número de semillas de plátano por año para cada ecosistema terrestre, teniendo presente la importancia de los registros de encuestas previas para el cálculo de cantidades de suministros y productos que permitieran determinar las hectáreas de cultivo.

- Producción y fabricación: en esta etapa se tuvo en cuenta la cosecha que sale de los cultivos. Los suministros estuvieron basados en fertilizantes, polietileno en bolsa, desinfectantes, bandas elásticas, fibras, y el combustible para el medio de transporte.

- Poscosecha: en el proceso se corta el plátano y se evidencia la expulsión de un líquido llamado látex, el cual se remueve con la aplicación de un champú. Es importante en este momento tener en cuenta el resultado final de la fruta vendida en cada hectárea por año.

- Distribución: se estima el cálculo del consumo de gasolina en la distancia recorrida, para cada agroecosistema.

\section{Resultados}

La metodología empleada en este trabajo se desarrolló buscando diferentes fuentes bibliográficas tanto dentro como fuera de Colombia. Posteriormente se realizó un cuestionario con el fin de determinar qué tanta aceptación puede tener el comprar productos biodegradables en la parte deportiva, al presentar como un plus del proceso la relevancia del cuidado del medio ambiente, bajando el nivel de contaminación sin que se afecte la calidad de los artículos. Para dicha encuesta se realizó una muestra aleatoria de 64 personas, entre los 16 y los 42 años de edad, teniendo en cuenta que es una población potencial de compra — son los rangos de existencia de mayor ejercitación-, con el fin de determinar los puntos de vista acerca de la reutilización de productos biodegradables en el ámbito deportivo.

Para identificar el conocimiento sobre las propiedades de la calceta de plátano para su reuso en la fabricación de elementos deportivos, se grafican los resultados arrojados por el cuestionario.

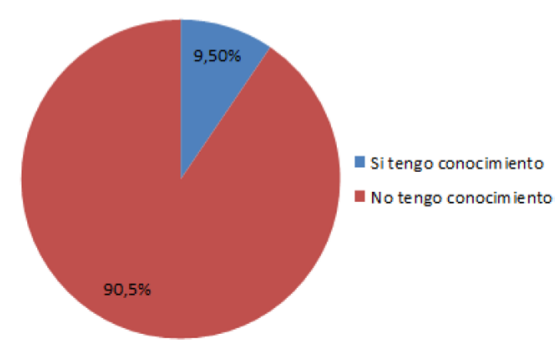

Figura 1. Conocimiento de los diferentes tipos de productos biodegradables con calceta de plátano.

Fuente: elaboración propia. 
La figura 1 muestra los resultados para la pregunta: ¿Sabía usted que con la calceta de plátano se pueden hacer diferentes tipos de productos biodegradables? Así, se observa que el $90,5 \%$ no tenía conocimiento y el $9,50 \%$ sí contaban con algún conocimiento.

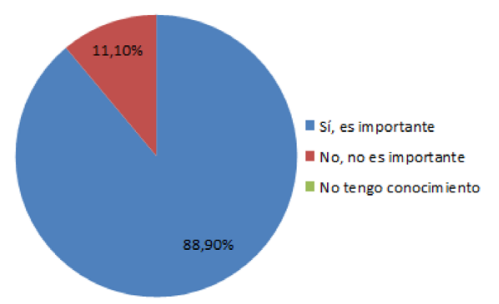

Figura 2. Importancia de aprovechar el residuo del plátano en productos nuevos. Fuente: elaboración propia.

La figura 2 muestra los resultados para la pregunta: ¿Considera importante hacer reuso de lo que no se aprovecha?, teniendo en cuenta que el $12 \%$ del fruto del plátano se aprovecha y que el $88 \%$ queda como residuo. El 88,9 \% de las personas encuestadas piensan que sí es importante y el $11,1 \%$ no tienen idea.

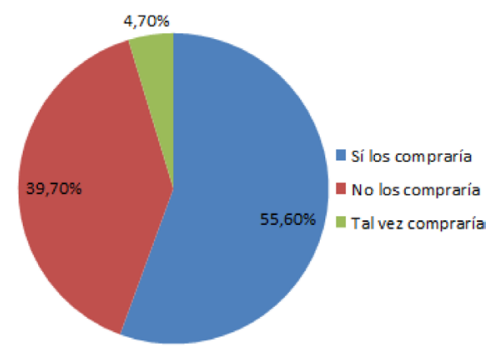

Figura 3. Compra de elementos deportivos hechos a base de calceta de plátano.

Fuente: elaboración propia.
La figura 3 muestra los resultados para la pregunta: ¿Compraría elementos deportivos hechos a base de calceta de plátano? Se obtuvo como respuesta que el 55,6\% sí los compraría; un 39,7 \%, tal vez compraría; y un 4,8\% no los compraría.

Para determinar el conocimiento sobre el nivel de aprovechamiento de productos elaborados con la calceta de plátano, se visualizan los resultados arrojados por el cuestionario.

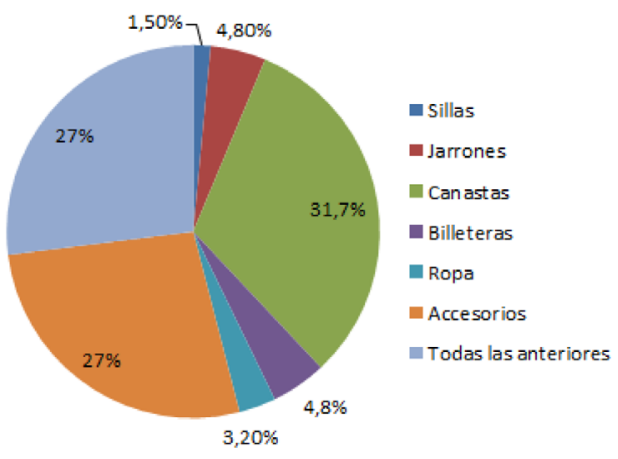

Figura 4. Productos que se pueden elaborar a partir del residuo de la calceta de plátano. Fuente: elaboración propia.

La figura 4 muestra los resultados para la pregunta: ¿Qué productos cree usted que se pueden elaborar a partir del residuo conocido como calceta de plátano? Los resultados fueron: el 31,7 \% escogió canastas, el $27 \%$ dijo que accesorios y otro 27 $\%$ eligió todas las anteriores, un $4,8 \%$ dijo billeteras y otro 4,8 \% jarrones, un 3,2\% escogió ropa y un $1,5 \%$ sillas. 


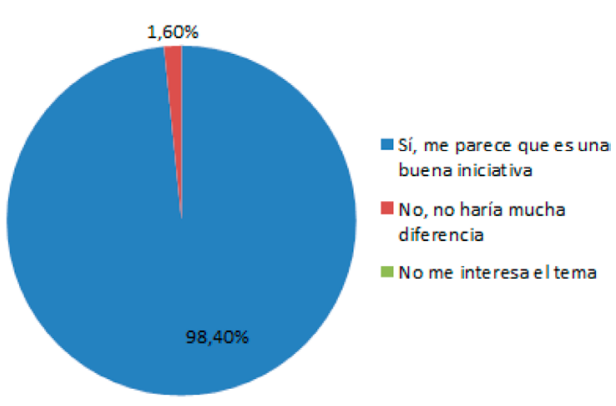

Figura 5. Perspectiva sobre el hecho de que las empresas trabajen con material biodegradable.

Fuente: elaboración propia.

La figura 5 muestra los resultados para la pregunta: ¿Le parece a usted que las empresas deben trabajar con material biodegradable en la producción de nuevos productos para cuidar el medio ambiente? En esta se encontró que el 98,4 \% sí le parece que es una buena iniciativa y un 1,6 $\%$ piensa que no haría mucha diferencia.

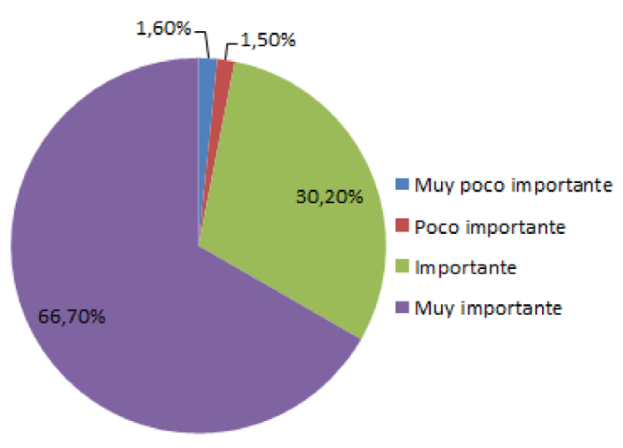

Figura 6. Importancia de concientizar a la gente sobre la compra de elementos biodegradables.

Fuente: elaboración propia.
La figura 6 muestra los resultados para la pregunta ¿Qué tan importante cree que es concientizar a la gente sobre la compra de elementos biodegradables? Se obtuvo como resultado un $66,7 \%$ para Muy importante; un 30,2 \%, Importante; y $1,6 \%$, Poco importante; $1,5 \%$, Muy poco importante.

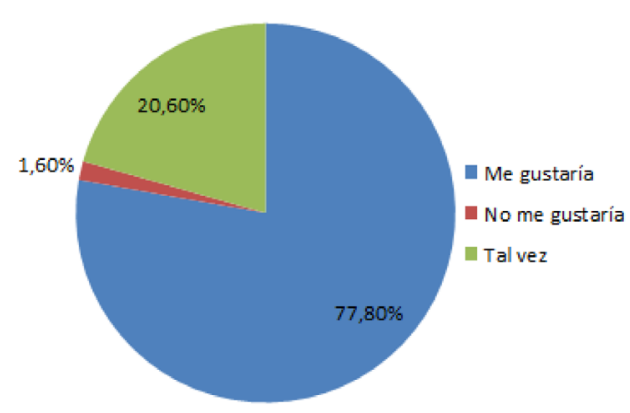

Figura 7. Preferencia para comprar elementos deportivos que disminuyan el uso de materiales hechos con bioplástico. Fuente: elaboración propia.

La figura 7 muestra los resultados para la pregunta: ¿Le gustaría comprar elementos deportivos de bajo costo que disminuyan el uso de materiales plásticos a base de petróleo? Las respuestas obtenidas fueron: un $77,8 \%$ para Me gustaría; un 20,6\%, Tal vez; y un $1,6 \%$, No me interesa.

\section{Discusión}

Dentro de la investigación se pudo evidenciar de manera relevante que tan solo un $9.5 \%$ de la población encuestada 
conoce la calceta de plátano como materia prima en la construcción de elementos biodegradables. Además, se vió que el 88 $\%$ considera que debe aprovecharse dicho material en la construcción de diferentes productos. Por otro lado, se demostró que el $55 \%$ le gustaría comprar elementos deportivos hechos con materiales amigables con el medioambiente. Asimismo, el $98.4 \%$ considera que este trabajo es una buena iniciativa para ser materializada y que tendría buena acogida.

Con los resultados obtenidos en la entrevista, se evidenció que un $88,9 \%$ de las personas encuestadas creen que sí es importante aprovechar el residuo de plátano en algún producto nuevo para generar un beneficio al medio ambiente, ya que el 12 $\%$ del fruto del plátano se aprovecha y el $88 \%$ queda como residuo, contrastado con Pedraza (2019), quien plantea la importancia de darle un uso a la calceta de plátano teniendo en cuenta la explotación del producto, al implementar el residuo en la fabricación de tejas de un material compuesto que se desarrolla y obtiene por medio de un método óptimo de extracción de la fibra de pseudotallo de plátano.

Al preguntar la opinión de las personas sobre si las empresas deberían trabajar con material biodegradable en la producción de nuevos productos, se obtuvo como resultado que al 98,4\% de la muestra encuestada sí le parece que es una buena iniciativa y un 1,6\% piensa que no haría mucha diferencia. Según Velastegui, et al., (2017) quien analiza el aprovechamiento del residuo del plátano para producir materiales plásticos biodegradables, muestra cómo los residuos orgánicos producidos en los cultivos plataneros no son aprovechados correctamente debido al miedo de los productores de implementar nuevas tecnologías porque piensan que este puede ser un factor que afecte la economía de su empresa. Sin embargo, esto se refuta teniendo en cuenta los resultados de la pregunta planteada, ya que muestra la aceptación de las personas por los productos hechos con material biodegradable para reemplazar el plástico y disminuir la contaminación ambiental.

\section{Conclusiones}

Por medio de esta investigación se identificaron las propiedades de la calceta de plátano, para usarla como materia prima en la fabricación de productos como estacas, conos, platillos, etc., lo que brinda una buena expectativa en la elaboración eficaz de elementos deportivos.

La encuesta realizada a los posibles clientes potenciales generó resultados 
favorables hacia la aceptación de dichos elementos, los cuales ocasionarían un buen impacto en el cuidado del medio ambiente, disminuirían la tasa de contaminación en las zonas productoras, evitarían el taponamiento de las tuberías y generarían una buena circulación del agua. Igualmente, los encuestados enfatizaron la relevancia para la utilización de productos ecoamigables con el ambiente y así mismo la disminución de productos altamente contaminantes.

El desarrollo permitió visualizar la importancia de dar uso a los residuos que, aunque sean orgánicos, pueden generar contaminación. De esta manera se aprovecha esta materia prima para crear un material nuevo y producir o fabricar elementos deportivos y reducir el uso del plástico.

Este artículo se desarrolla en el ámbito ambiental y deportivo. Por lo tanto, como muchos otros proyectos ambientalistas y ecológicos que se han llevado a cabo, tiene la facilidad de adaptarse a las nuevas tendencias que se han impuesto en los últimos tiempos, ya sea porque es importante o necesario para que el cambio climático y la contaminación disminuyan.

\section{Referencias}

Alarcón, M., Berrocal, E., Canales, V., Ipanaqué, J., y Jaimes, R. (2019). Producción y comercialización de platos descartables y biodegradables hechos a base de papel kraft y hojas de plátano.Universidad San Ignacio de Loyola http://repositorio.usil. edu.pe/handle/USIL/9881

Pedraza, C. (2019). Caracterización de la fibra del pseudotallo de plátano como refuerzo y desarrollo de un material compuesto para fabricación de tejas. Universidad Pedagógica y Tecnológica de Colombia, Duitama https://repositorio.uptc.edu.co/ bitstream/001/2768/1/TGT_1401.pdf

Rodríguez, A., Rodríguez, C., Gutiérrez, S. (2017). Memorias de oficio: calceta de plátano Cereté (Colombia). CENDAR. https://cendar-repositorio.metabiblioteca. org/handle/001/3971

Valenzuela, E. Castañeda, D. y Cano, N. (2019). Determination of Plantain Crops Associated with Coffee Environmental Impacts on Agroecosystems by Means of Life Cycle Assessment: Case Study in the Southwest of Antioquia (Colombia) .DYNA (Colombia), 86(211), 112-121. https://www-scopuscom.proxy.bidig.areandina.edu.co/record/ display.uri?eid=2-s2.0-85084412052\&origin $=$ results list $\&$ sort $=$ plf $-\mathrm{f} \& \mathrm{~s}-$ $\mathrm{rc}=\mathrm{s} \& \mathrm{st} 1=$ platano $\& \mathrm{st} 2=\& \mathrm{sid}=6 \mathrm{~b} 827 \mathrm{acf}-$ $6098 \mathrm{db} 7 \mathrm{fe} 14 \mathrm{de} 94 \mathrm{fb} 070 \mathrm{c} 045 \&$ sot=b\&sd$\mathrm{t}=\mathrm{b} \& \mathrm{sl}=22 \& \mathrm{~s}=$ TITLE-ABS-KEY\%28platano $\% 29 \&$ relpos $=10 \&$ citeCnt $=0 \&$ searchTerm=

Velasteguí, A., Arévalo, A. y Bloisse, S. (2017). Análisis sobre el aprovechamiento de los residuos del plátano, como materia prima para la producción de materiales plásticos biodegradables. Dominio de las Ciencias, 3(2), 506-525. https://dialnet.unirioja.es/ servlet $/$ articulo? codigo $=6325873$ 